\title{
Numerical simulation of cooling electronic components mounted in a vertical wall by natural convection
}

\author{
KARIM LAHMER ${ }^{\mathrm{a}}$ AND RACHID BEsSAÏH \\ Université Constantine 1, Laboratoire d'Energétique Appliquée et de Pollution LEAP, Faculté des Sciences de la Technologie, \\ Route d'Ain El Bey, 25000 Constantine, Algeria
}

Received 30 January 2013, Accepted 17 January 2014

\begin{abstract}
This paper is a numerical work to investigate thermal interactions between heat fluxes generated by electronic devices mounted on a vertical printed circuit board (PCB). In fact, the effect of some parameters such as Grashof number $(G r)$, the distance $(S)$ between the heat sources and the upper exit distance $\left(L_{\mathrm{e}}\right)$ was studied. The results show that a regular and uniform heat sources distribution in the inlet is very important in order to take advantage from the leading edge of the boundary layer and to obtain the necessary heat dissipation. The impact of parameters on the heat dissipation, characterized by the Nusselt number, has a different importance. For a Prandtl number $\operatorname{Pr}=0.71$, the $(G r)$ increases the heat exchange that is reflected by the increase of Nusselt number, and also participates to the formation of recirculation zones. The total Nusselt number $\mathrm{Nu}$ is increased when $(G r)$ is multiplied by $10^{2}$. With regards to the distance $(S)$ between the heat sources, the results show that Nu increases about $7 \%$ if the distance $(S)$ doubles, and becomes approximately $17.8 \%$ when $(S)$ quadruples. At the end, the heat transfer increases when increasing the distance $\left(L_{\mathrm{e}}\right)$ of the upper exit length, especially on the second component. The total Nusselt number increases by $1.6 \%$ when $\left(L_{\mathrm{e}}\right)$ increases by about $67 \%$.
\end{abstract}

Key words: Natural convection / vertical adiabatic plate / cooling electronic components / numerical simulation / finite volumes

\section{Introduction}

This work has its applications in electronic equipment cooling, thin films manufacturing, and chemical vapors decomposition. Understanding the characteristics of the flow and generated heat transfer allow an optimal management of heat transfer for design. A study of the US Air Force has indicated that over $50 \%$ of electronic systems defects are closely related to the uncontrolled rise in temperature, Yeh [1].This explains why many numerical and experimental studies have been performed to resolve this problem. Dehghan and Behnia [2] have carried out a numerical study on natural convection cooling of two configurations of heat sources, immersed in parallel series of cavities containing coolant (CF-72). In addition, two thermal conductivities (low and high) were considered. Wang et al. [3] performed a numerical study on the cooling of five integrated circuits mounted on an adiabatic vertical plate. By varying their size, the authors sought to determine the effect of a partial blockage of the flow of heat transfer. Ramón and Berbakow [4] conducted a numerical study of natural convection cooling of heat

\footnotetext{
a Corresponding author: lahmer.karim@sonelgaz.dz
}

sources mounted on one of the vertical walls of a cube array.

The results obtained show that the flow fluctuates with the Rayleigh number. Dias and Milan [5] compared the two and three dimensional methods in order to understand both the physical phenomena associated with this type of configuration (vertical plate equipped with heat sources), and the literature. Da Silva et al. [6] investigated a vertical flat plate containing heat sources distributed in two classes of geometries: the first contains heat sources with fixed dimensions; and the second contains a single heat source with variable dimensions. The results indicate that in an open channel, the optimal space between the heat sources increases with the Rayleigh number. Also, the position and size of a single source with a fixed heat flow can be optimized for maximum overall thermal performance. Comunelo and Guth [7] conducted a comparison between experiment and numerical simulation. The configuration studied is an enclosure containing five transducers mounted vertically on a plate, by varying the geometrical parameters of the obtained configurations, an increase in the heat transfer coefficient is observed. Bhowmic and $\mathrm{Tu}[8]$ studied experimentally the natural 


\section{Nomenclature}

\begin{tabular}{|ll|}
\hline$A$ & Exposed area of heated sources \\
$g$ & Acceleration due to gravity, $\mathrm{m}^{-2}$ \\
$G r$ & Grashof number $G r=\frac{g \beta\left(T_{S}-T_{0}\right)}{v_{\text {air }}^{2}} L_{S}^{3}$ \\
$L_{\mathrm{e}}$ & Output length downstream of the second electronic component, $\mathrm{m}$ \\
$L_{\mathrm{i}}$ & Input length before the first electronic component, $\mathrm{m}$ \\
$L_{S}$ & Characteristic length of the electronic component, $\mathrm{m}$ \\
$L_{x}, L_{\mathrm{y}}$ & Dimensions of the computational domain, $\mathrm{m}$ \\
$N u_{\text {Local }}$ & Local Nusselt number, $=\left.\frac{\partial \theta}{\partial \mathrm{n}}\right|_{\mathrm{A}}$ \\
$N u$ & Total Nusselt number, $=\int_{A} N u \mathrm{~d} A$ \\
$N u s_{\mathrm{s} 1, \mathrm{~s} 2}$ & Total local Nusselt number for source 1 and for source 2 \\
$n$ & Normal coordinates \\
$P$ & Pressure, Pa \\
$P r$ & Prandtl number Pr $=\frac{v_{\text {air }}}{\alpha_{\mathrm{air}}}$ \\
$M_{\mathrm{in}-\text { out }}$ & Difference between mass inflow and outflow rates, $\mathrm{m}^{3} \cdot \mathrm{s}^{-1}$ \\
$S$ & Distance between the electronic components, $\mathrm{m}$ \\
$T_{0}$ & Ambient temperature, $\mathrm{K}$ \\
$T_{S}$ & Maximum temperature of each electronic component, $\mathrm{K}$ \\
$U, V$ & Horizontal and vertical dimensionless velocities \\
$X, Y$ & Dimensionless coordinates \\
$\mathrm{Greek}$ letters & \\
$\alpha$ & Thermal diffusivity, $\mathrm{m}^{2} \cdot \mathrm{s}^{-1}$ \\
$\beta$ & Coefficient of thermal expansion $=-\frac{1}{\rho}\left(\frac{\rho-\rho_{0}}{T-T_{0}}\right), \mathrm{K}^{-1}$ \\
$\lambda$ & Conductivity, W.m ${ }^{-1} \cdot \mathrm{K}^{-1}$ \\
$\theta$ & Dimensionless temperature $=\frac{T-T_{0}}{T_{S}-T_{0}}$ \\
$\varepsilon$ & Residuals \\
$\nu$ & Dimensionless kinematic viscosity, \\
$\rho$ & Density of fluid, kg.m ${ }^{-3}$ \\
$\rho_{0}$ & Dluid density at ambient condition, kg.m ${ }^{-3}$ \\
& Dimensionless stream function \\
\hline
\end{tabular}

convection cooling of four heat sources mounted in a vertical rectangular channel. The results show that the heat transfer coefficient is strongly affected by the number of heat sources; and if the height of the component is important, the heat transfer is much greater. Icoz and Jaluria [9] performed a numerical study of two-dimensional natural convection in a rectangular opened channel, containing two identical heat sources.

The authors studied in detail the behavior of heat transfer and the trajectory of air particles. The results show that the $(G r)$ and the distance between sources $(S)$, are the main parameters that affect the heat dissipation rate. Nadar [10] compared experimentally the horizontal arrangement towards the vertical heat sources and for different Rayleigh numbers $R a$ and sizes. The results show that when the length of the heat source is important, the Nusselt number increases. Also, when $R a$ increases, $\mathrm{Nu}$ increases for every geometric disposition. Harvest et al. [11] studied the thermal interactions as a consideration used in compact electronic devices. Their results show that if two heat sources are unevenly heated, the thermal power of one will affect the power of the other in certain limit of space. The objective of this work is to determine the impact of certain parameters to obtain an optimal distribution of the electronic components. We started with the effect of Grashof number $(G r)$ on heat dissipation, which is used to determine the threshold for convection changes from laminar to the turbulent regime. Then, the effect of the distance $(S)$ between the electronic components, and finally the effect of distance $\left(L_{\mathrm{e}}\right)$ downstream of the second component, were studied in order to design an installation that meets our expectations in terms of heat transfer.

\section{Geometry and mathematical model}

The geometry of the considered problem is shown in Figure 1. It consists of two identical electronic components (heat source) simulating two microprocessors mounted on a vertical adiabatic plate with $L_{x} \times L_{\mathrm{y}}$ dimensions. The two components have an identical length and height, $L_{S}$ and are separated by a distance, $S$. The distance between the lower free surface and the first component is the one between $L_{\mathrm{i}}$ and the second component and the upper free surface. Air enters through the lower cross-sectional area, and through the longitudinal surface drains right through the upper horizontal surface. The 


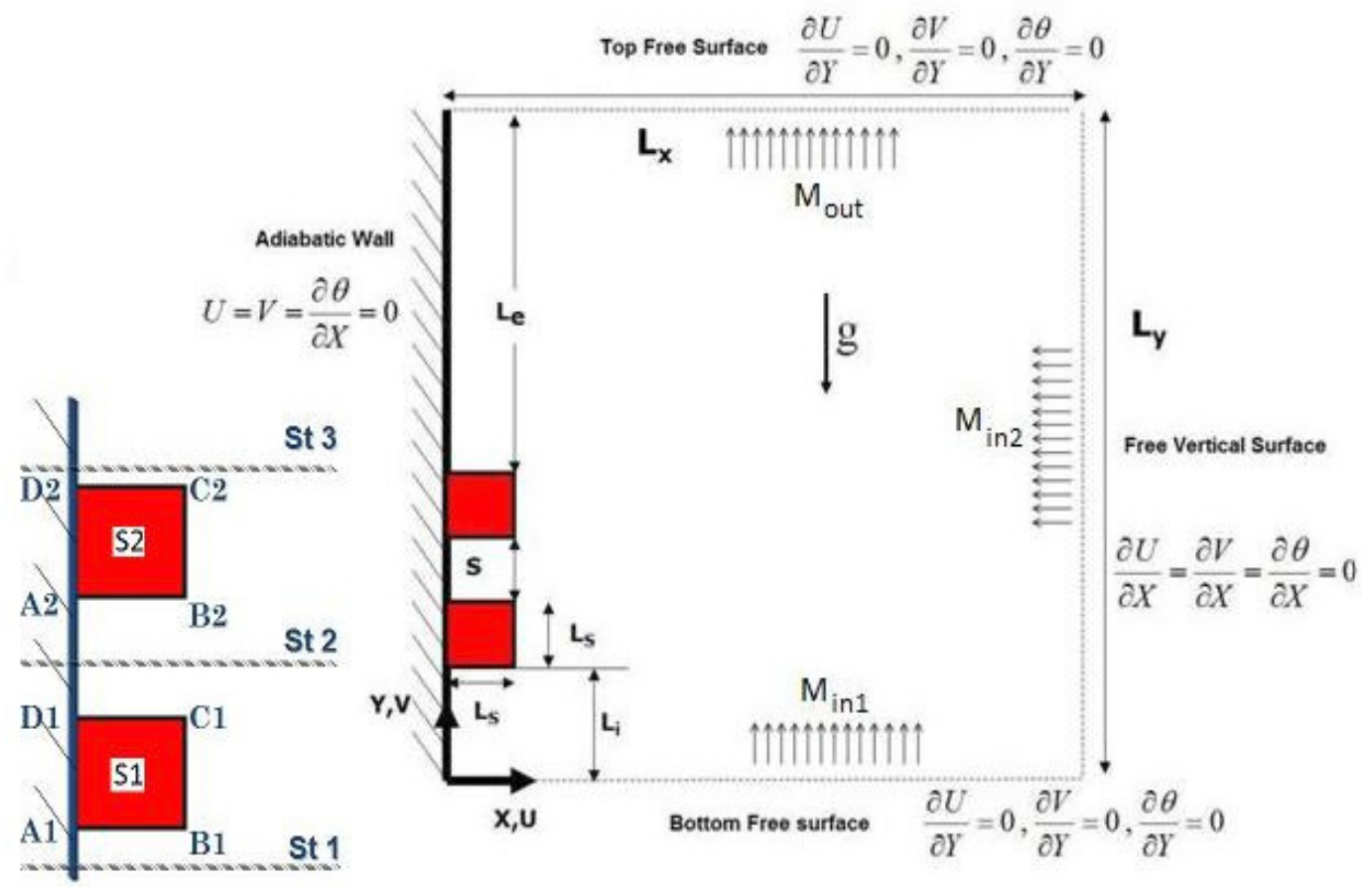

Fig. 1. Geometry and boundary conditions.

heat sources are maintained to the maximum temperature $T_{S}$ in the ambient temperature $T_{0}$, heat transfer through radiation has not been taken into consideration. Also, we considered that the flow is laminar, the fluid is Newtonian and incompressible, the viscous dissipation is negligible and the Boussinesq approximation is valid everywhere [12]. In order to reduce the number of physical parameters, the following characteristic expressions and dimensionless variables have been used:

- $L_{s}$ : characteristic length;

$-\frac{\alpha_{\text {air }}}{L_{S}}:$ characteristic velocity;

- $\rho_{0}\left(\frac{\alpha_{\text {air }}}{L_{S}}\right)^{2}:$ characteristic pressure;

- $X=x / L_{S} ; Y=y / L_{S} ; U=u\left(\alpha_{\text {air }} / L_{S}\right)^{-1} ; V=v$. $\left(\alpha_{\text {air }} / L_{S}\right)^{-1} ; \lambda=\lambda / \lambda_{\text {air }} ; V=\nu / \nu_{\text {air }} ;$

$$
\begin{aligned}
& P=\left(p-p_{0}\right) \frac{L_{S}^{2}}{\rho_{0} \alpha_{\text {air }}^{2}} \\
& \theta=\frac{T-T_{0}}{T_{S}-T_{0}}
\end{aligned}
$$

The new obtained dimensionless system of equations for the unsteady two-dimensional case becomes:

\section{Mass equation}

$$
\frac{\partial U}{\partial X}+\frac{\partial V}{\partial Y}=0
$$

\section{$X$-Momentum equation}

$$
\begin{aligned}
\frac{\partial(U U)}{\partial X}+\frac{\partial(V U)}{\partial Y}= & -\frac{\partial P}{\partial X} \\
& +\operatorname{Pr}\left\{\frac{\partial}{\partial X}\left[\frac{\partial U}{\partial X}\right]+\frac{\partial}{\partial Y}\left[\frac{\partial U}{\partial Y}\right]\right\}
\end{aligned}
$$

\section{$Y$-Momentum equation}

$$
\begin{aligned}
\frac{\partial(U V)}{\partial X}+\frac{\partial(V V)}{\partial Y}= & -\frac{\partial P}{\partial Y}+\operatorname{Pr}\left\{\frac{\partial}{\partial X}\left[\frac{\partial V}{\partial X}\right]\right. \\
& \left.+\frac{\partial}{\partial Y}\left[\frac{\partial V}{\partial Y}\right]\right\}+G r \operatorname{Pr}^{2} \theta
\end{aligned}
$$

\section{Energy equation}

$$
\frac{\partial(U \theta)}{\partial X}+\frac{\partial(V \theta)}{\partial Y}=\frac{\partial}{\partial X}\left[\frac{\partial \theta}{\partial X}\right]+\frac{\partial}{\partial Y}\left[\frac{\partial \theta}{\partial Y}\right]
$$

The following dimensionless boundary conditions are considered:

- at $X=0$ (adiabatic wall)

$$
U=V=0 ; \frac{\partial \theta}{\partial X}=0
$$

- at $X=L_{x} / L_{S}$ (free vertical surface)

$$
\frac{\partial U}{\partial X}=\frac{\partial V}{\partial X}=0 \frac{\partial \theta}{\partial X}=0
$$


Table 1. Grid effect on numerical solution.

\begin{tabular}{lcccc}
\hline \multicolumn{5}{c}{ Grids } \\
\hline & $G_{1}=42 \times 122$ nodes & $G_{2}=62 \times 222$ nodes & $G_{3}=72 \times 322$ nodes & $G_{4}=82 \times 422$ nodes \\
\hline$N u_{S 1}$ & 4.917493 & 4.255175 & 3.904676 & 3.768118 \\
$N u_{S 2}$ & 3.245160 & 2.970387 & 2.988137 & 3.004394 \\
$U_{\max }$ & 40.908160 & 50.349330 & 50.409930 & 50.4513 \\
$V_{\max }$ & 102.8202 & 101.6222 & 101.2285 & 101.0121 \\
$M_{\text {out }}-M_{\text {in }}$ & 0.0164 & 0.02299 & 0.02928 & 0.03474 \\
\hline
\end{tabular}
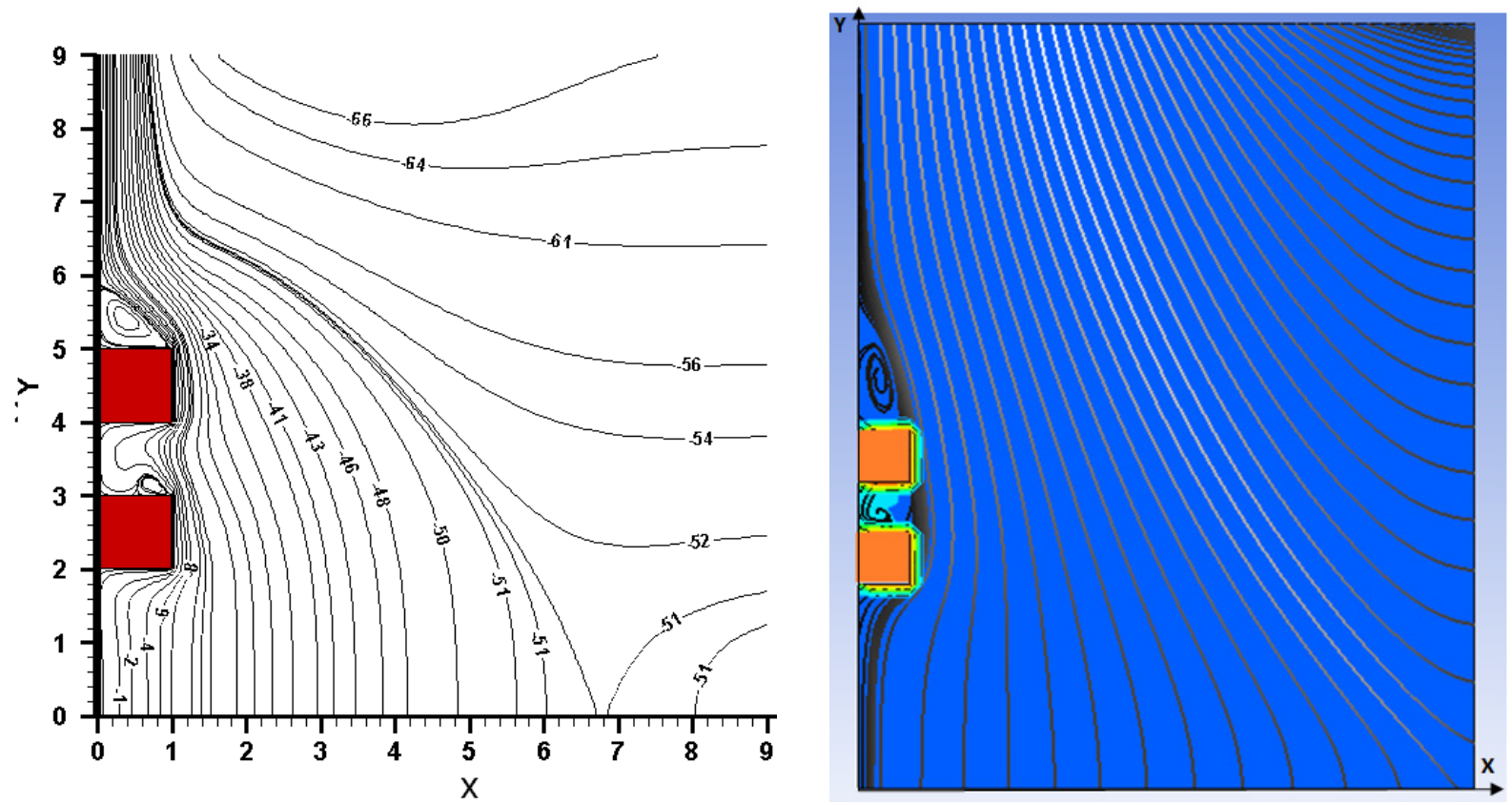

Fig. 2. Streamlines profiles in the study domain when $S=L_{s}$ and $G r=10^{4}$ (with our Fortran code at left and with FLUENT at right).

- at $Y=0$ (bottom free surface)

$$
\frac{\partial U}{\partial Y}=0 \quad \frac{\partial V}{\partial Y}=0 \quad \frac{\partial \theta}{\partial Y}=0
$$

- at $Y=L_{\mathrm{y}} / L_{S}($ top free surface $)$

$$
\frac{\partial U}{\partial Y}=0 ; \quad \frac{\partial V}{\partial Y}=0 \quad \frac{\partial \theta}{\partial Y}=0
$$

\section{Numerical method}

The finite volume method was used to discretize the dimensionless equations system of the mathematical model. They have been implemented in a FORTRAN code. The scalar quantities are stored at the control volumes centers, while the velocity components $(U$ and $V$ ) are located on the sides. The SIMPLER algorithm [13] was used to handle the pressure-velocity coupling. The discretized equations were solved iteratively in each direction along the axes, using the TDMA algorithm. To solve these equations, we have undertaken calculations on four types of mesh.
In order to know grid influence on numerical solution we adopted the same parameters values $(\operatorname{Pr}=0.71$; $\left.G r=10^{4} ; S=L_{s}\right)$ on these four different meshes (Tab. 1). After simulations, the obtained results are a difference that doesn't exceed $5 \%$ between the grids $\mathrm{G}_{3}$ and $\mathrm{G}_{4}$. Consequently, we opted for the $\mathrm{G}_{3}$ mesh in order to optimize the CPU time and the cost of computations.

To obtain a convergent solution, we defined the followed criterion:

$$
\begin{aligned}
& \left|\frac{\theta^{n+1}-\theta^{n}}{\theta^{n}}\right|_{\max } \leqslant \varepsilon ; \quad\left|\frac{U^{n+1}-U^{n}}{U^{n}}\right|_{\max } \leqslant \varepsilon ; \\
& \left|\frac{V^{n+1}-V^{n}}{V^{n}}\right|_{\max } \leqslant \varepsilon
\end{aligned}
$$

The criterion is satisfied when the relative error between two successive iterations in all nodes is lower than $\varepsilon=10^{-6}$.

Finally, (Fig. 2) describes the performance of developed code which has been validated by comparison with a coupled and pseudo transient laminar flow realized by FLUENT. 

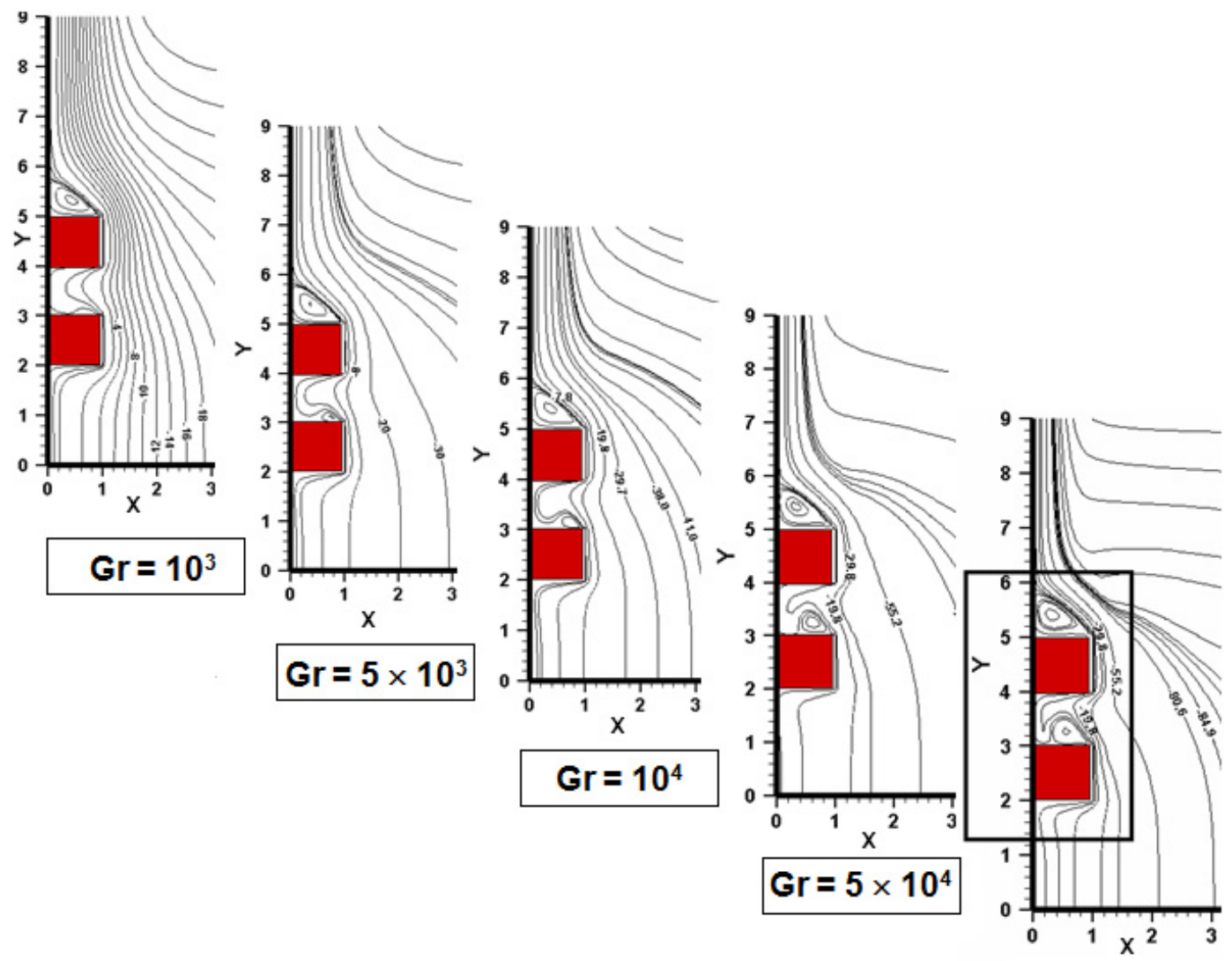

$\mathrm{Gr}=10^{5}$

Fig. 3. Streamlines profiles for different values of $(G r)$.

\section{Results and discussion}

\subsection{Effect of Grashof number (Gr)}

The parameters variation of $L_{S}$ and $\Delta T$ create different values of the Grashof number. The considered ones are: $G r=10^{3}, 5 \times 10^{3}, 10^{4}, 5 \times 10^{4}$ and $10^{5}$ in order to determine its influence on the velocity profiles, temperature fields contours, and the Nusselt number. The geometric data taken for this study are: $S=L_{S}, L_{\mathrm{e}}=4 \times L_{S}$ and $L_{\mathrm{i}}=2 \times L_{S}$, with respect to the flow lines described by the stream function $\psi(V=-\partial \psi / \partial X ; U=\partial \psi / \partial Y)$; (Fig. 3). They show in one hand, their deviation when they are in contact with electronic components, and in other hand, the formation of vortex zones with various sizes, mostly in the downstream regions of the heat sources. They are much smaller on the face C1-D1, the first component for low values of $\operatorname{Gr}\left(10^{3} \leqslant G r<5 \times 10^{3}\right)$, then gradually becomes larger when $G r$ number increases, until it reaches a maximum size near the solid plate wall when $G r=10^{5}$ (Fig. 4 - zoom of rectangle for $G r=10^{5}$ ). Near the lower free surface, we find that the increase in Gr increases the mixing of two air flows inward in the field. Therefore, the effect of a gradual increase of the Grashof number of $10^{3}$ to $10^{5}$ will result in an increase of Archimede forces

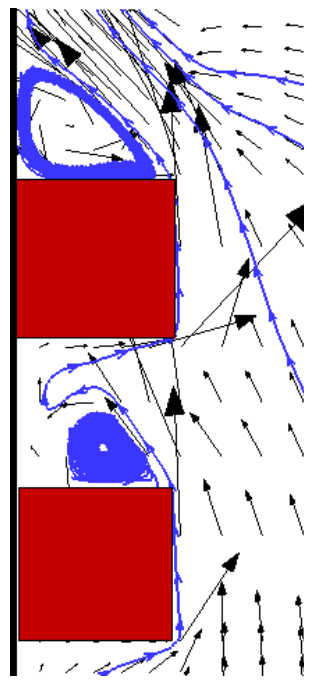

Fig. 4. Zoom of the rectangular zone of Figure 3 describing velocity vectors and vortex for $G r=10^{5}$.

(buoyancy force), which gives stratified and lengthen flow lines along the vertical solid wall.

By observing the temperature fields (Fig. 5), we distinguish isotherms close to each other near the 

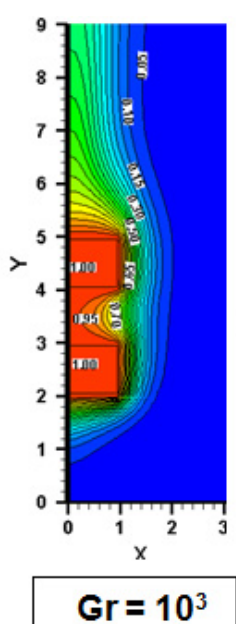

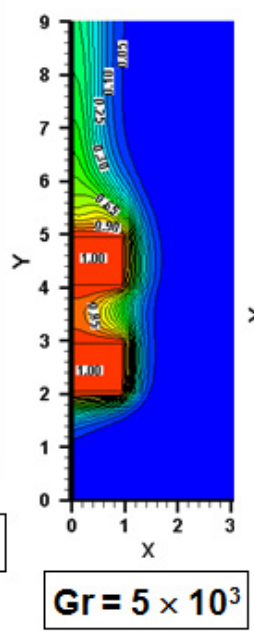

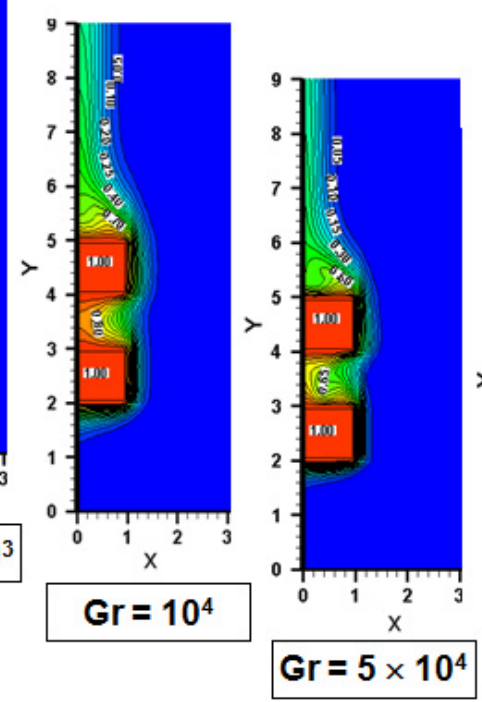

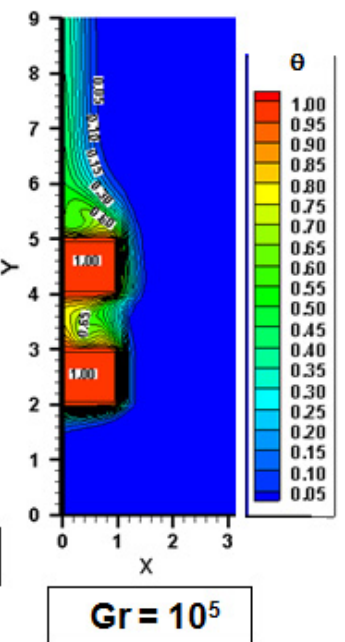

Fig. 5. Isotherms contours for different values of Grashof number $(G r)$.

Note: The maximum temperature is located on each heat source.

components, showing a significant exchange of heat from other parts of domain regions. In fact, on the electronic components the temperature is on the maximum, then, gradually regresses away from them. The Grashof number effect is very important because of the isotherms distortion, especially on the faces of the components that are perpendicular to the wall of the solid plate (C1-D1 and $\mathrm{C} 2-\mathrm{D} 2$ ), and that denounces the presence of vortex (recirculation zones). The gradual increase in the number of Grashof increases the heat dissipation, especially distinct in the space between the components and the downstream of the second component. This is due to the important decrease of fluid temperature in these two regions, which promotes the removal of heat. The creation of recirculation zones in the downstream region of components creates a significant change in the thermal field and favors an optimal heat transfer.

Concerning the vertical velocity function curves $V=$ $f(X)$ at different stations (Fig. 6), we know that the velocity is zero on the wall as well as components and maximum when $L_{S}<X<2 \times L_{S}$ or above the surfaces (B1-C1and B2-C2), then gradually fades beyond it. When comparing the maximum values of velocity between $G r=10^{3}$ and $G r=10^{5}$ at different median stations an increase is reported in the velocity value by 4.7 at the first station (upstream of the first component), by 8.26 to the second station (between the two components) and finally by 7.6 to the third station (downstream of the second component). However, the velocity values reach their maximum level at the third station. Sometimes negative values of vertical velocity indicate the reverse flow direction explaining the formation of large cells in which the air is entrapped within the recirculation zone.
The local Nusselt number as a function of Grashof $N u_{\text {Local }}$ on all six sides of the electronic components (Fig. 7) shows curves almost stratified, except on the faces C1-D1 and C2-D2, on which the curves become parables to $N u_{\text {Local }}$ values of $\left(5 \times 10^{4} \leqslant G r<10^{5}\right)$, reflecting a sudden drop in the rate of heat transfer. On the faces B1-C1 and B2-C2, we note that when $\left(5 \times 10^{4} \leqslant G r<\right.$ $10^{5}$ ), there is an increase in local Nusselt number until it reaches a maximum value, followed by a change in the hyperbole profiles that results in significant heat dissipation (close to the B1 and B2 points). One can also note that the $N u_{\text {Local }}$ profiles for the first component are higher than the ones of the second one because it directly benefits from contact with the start of the thermal boundary layer. Meanwhile, the maximum value of $N u_{\text {Local }}$ is reached in lower vertical surface, between B1 and $\mathrm{C} 1$ points. In short, the increase of Grashof amplifies the heat exchange resulting in an increase of the Nusselt number, and also participates in the formation of vortex zones downstream of each source. Our results reflect an increase in the overall total Nusselt number (Fig. 8), which triples when $\mathrm{Gr}$ is multiplied by $10^{2}$.

\subsection{Effect of the distance $S$ between the electronic components}

The distance $(S)$ between the electronic components is one of the geometrical factors that impact the behavior of the fluid within the area studied as well as the corresponding heat transfer. In order to determine this impact, we varied the distance between the two sources of heat, assuming $S=0.5 \times L_{S}, S=1 \times L_{S}$ and $S=2 \times L_{S}$ and 


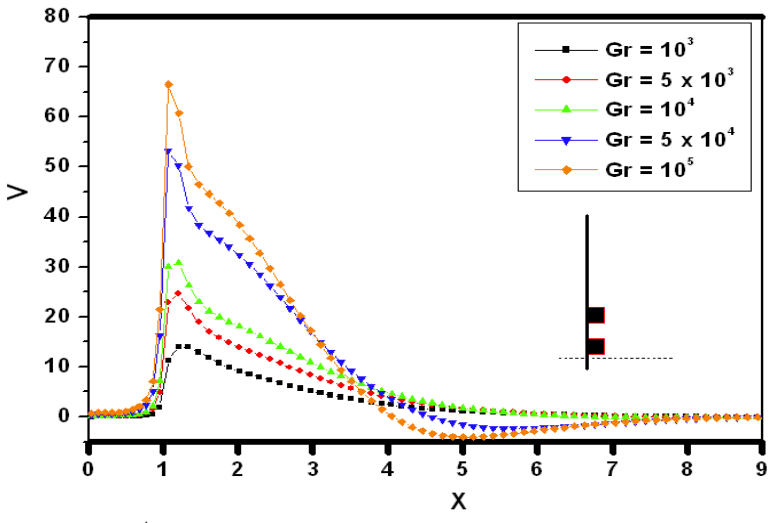

$1^{\text {st }}$ station: Before the first component

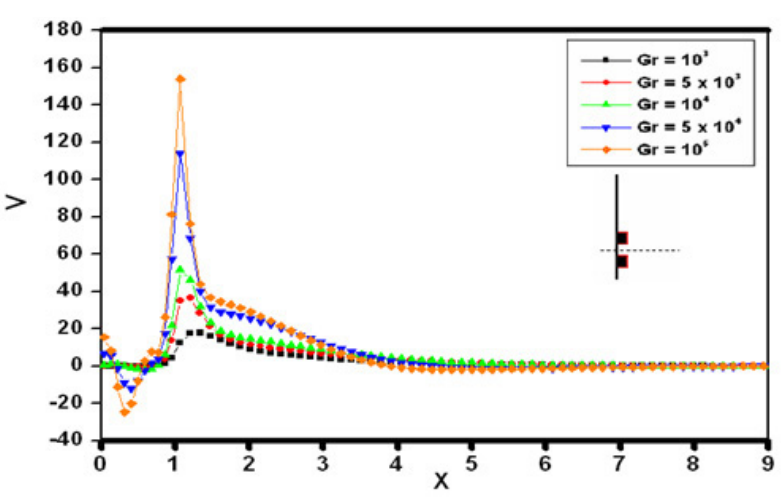

$2^{\text {nd }}$ station: Between the two components

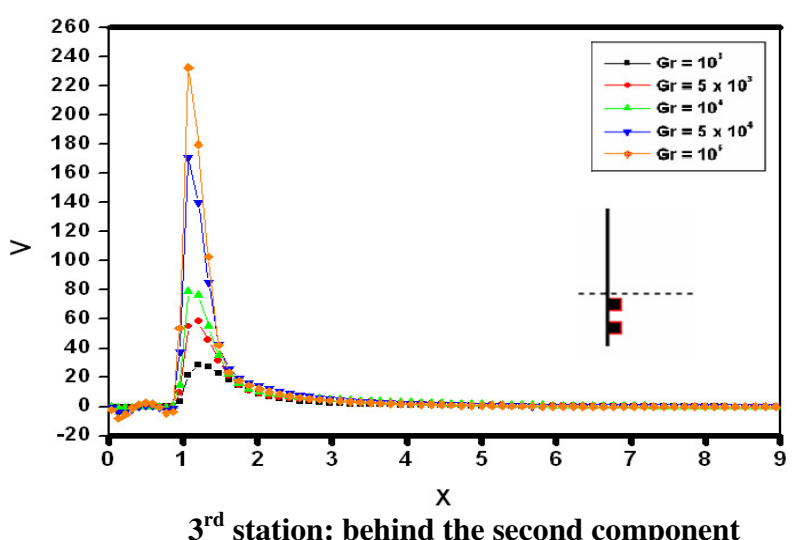

Fig. 6. Velocities profiles with Grashof number at different stations with $S=L_{s}$ and $L_{\mathrm{e}}=4 \times L_{s}$.

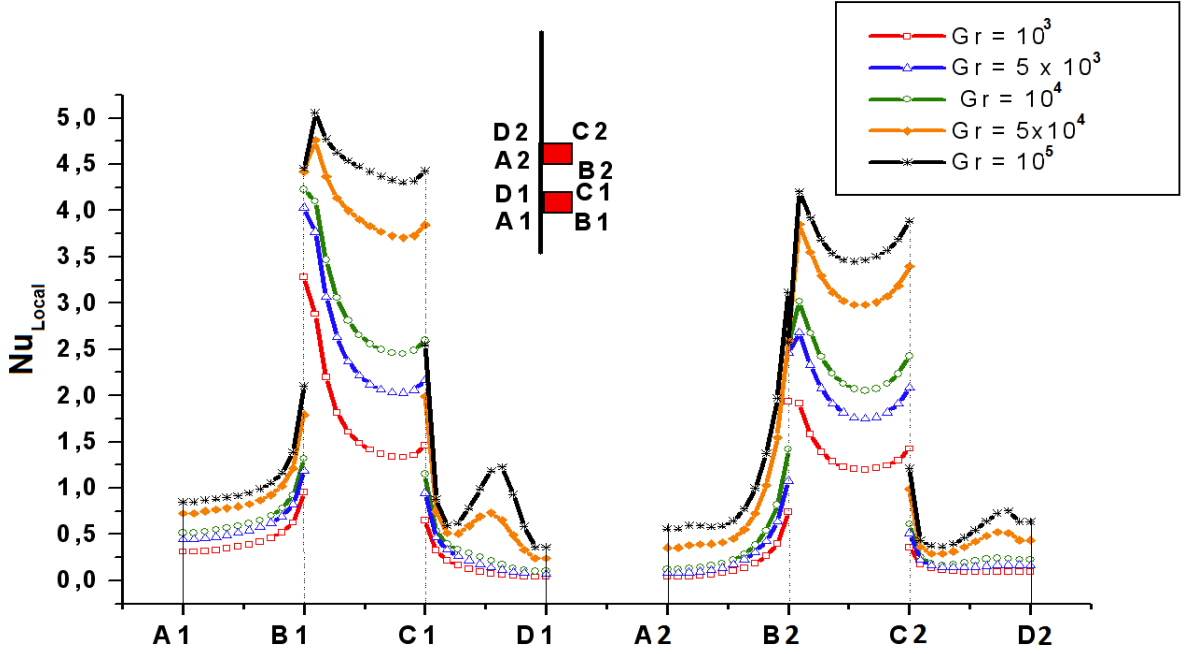

Fig. 7. Variations of $\left(N u_{\text {Local }}\right)$ according to different values of $(G r)$ when $S=L_{s}$ and $L_{\mathrm{e}}=4 \times L_{S}$.

$G r=10^{4}$. The results of the stream functions (Fig. 9) show an almost regular line, as well as the formation of recirculation zones, some of small-scale localized between the two components or downstream of the surface C1-D1 of the first component, a second zone more consistent in terms of scale is located downstream of the surface C2-D2 of the second component. When $S=2 \times L_{S}$, the flow lines regularity is less pronounced, and recirculation zones are larger and reach the wall of the plate. This demonstrates that the increase of heat transfer in this place is caused by the accumulation of the mixture of currents sweeping the two components simultaneously.

The scope of the isotherms shown in Figure 10, indicates the increase of heat transfer (reduced temperature) recorded in the case of large spacing between the 


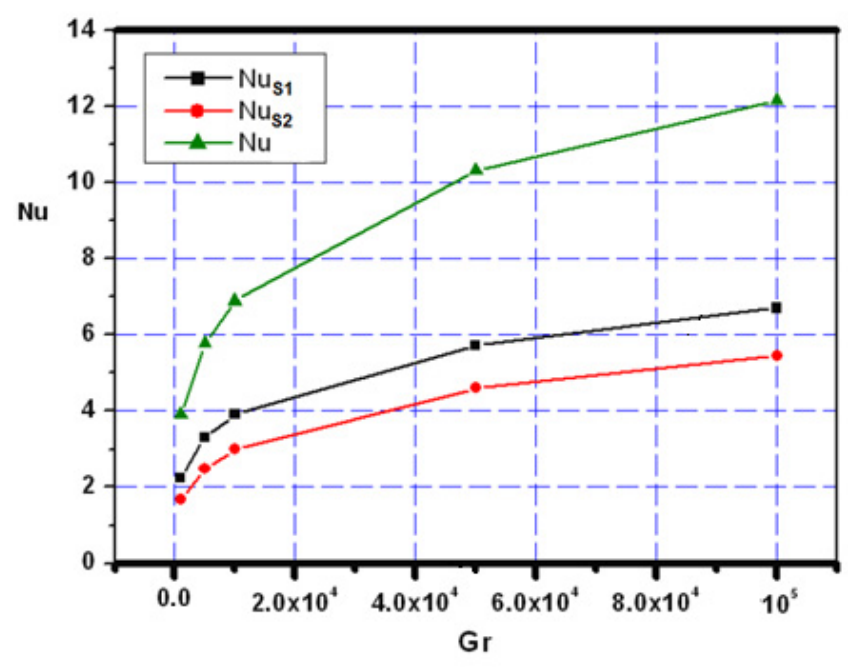

Fig. 8. Variation of total Nusselt number $(N u)$ with the different $(G r)$ values.

Note: $N u_{S 1}$ and $N u_{S 2}$ are respectively the total Nusselt number of the first and the second heat sources, when $S=L_{S}$ and $L_{e}=4 \times L_{S}$.
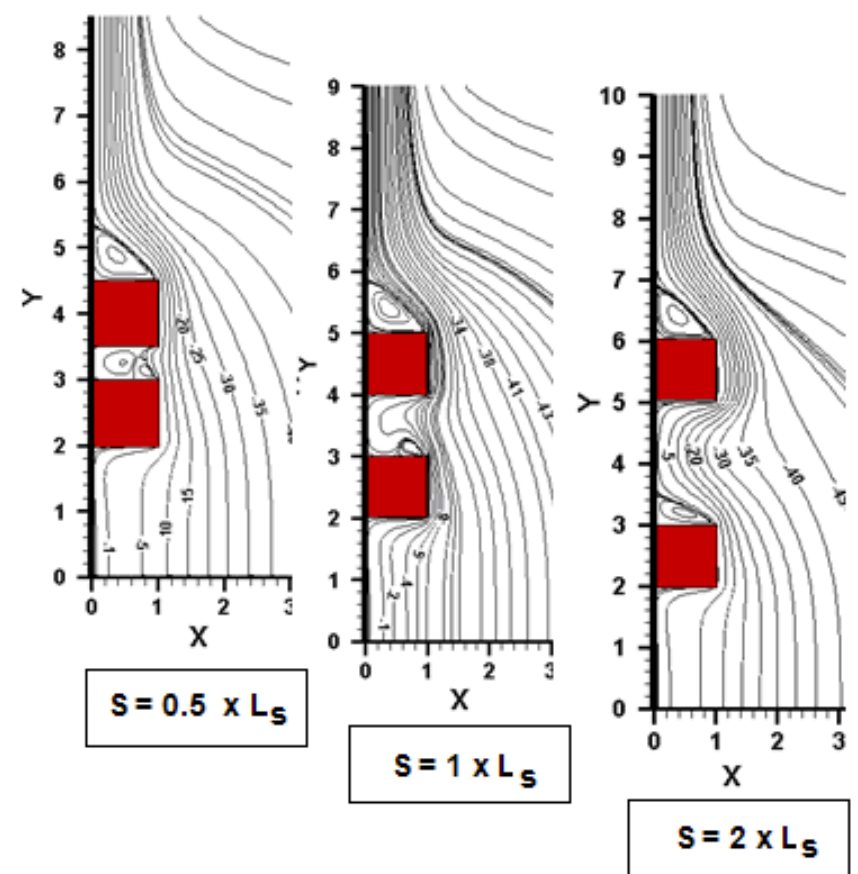

Fig. 9. Streamlines profiles $\psi$ for different values of the distance $(S)$ between the two heat sources when $G r=10^{4}$.

components, especially when considering temperature intensity between heat sources.

By varying the value of the distance $(S)$ and determining the local Nusselt number on the three sides of the first component in contact with the air, we detect the removal rate of heat at these surfaces. Indeed, on three sides (Fig. 11a), we find a quantity of heat removed on the same face A1-B1 for three values of $(S)$ considered, something that can also be seen on the face B1-C1, except
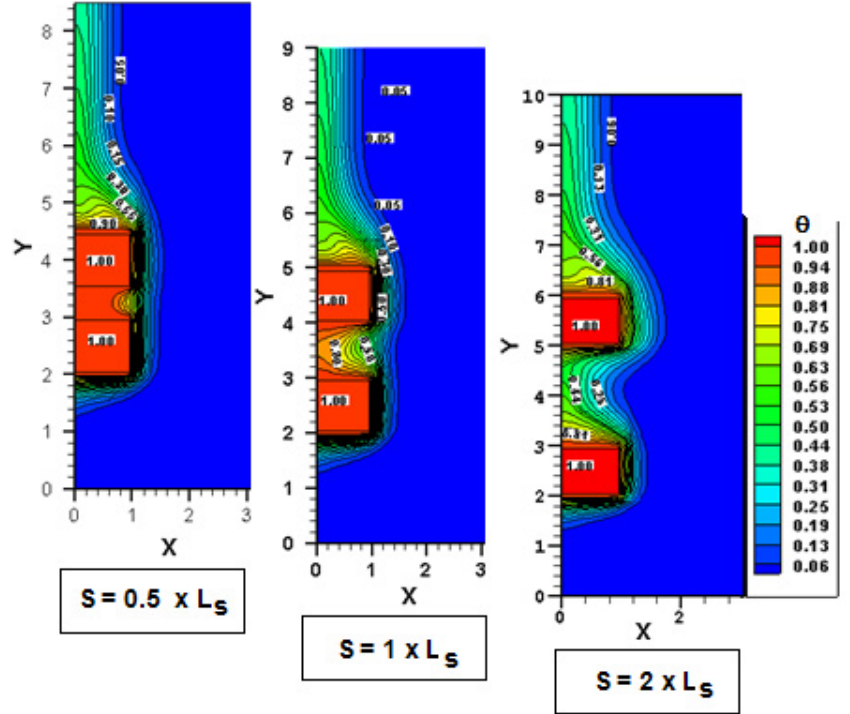

Fig. 10. Isotherms contours with different values of the distance $(S)$ between the two sources of heat for $G r=10^{4}$.

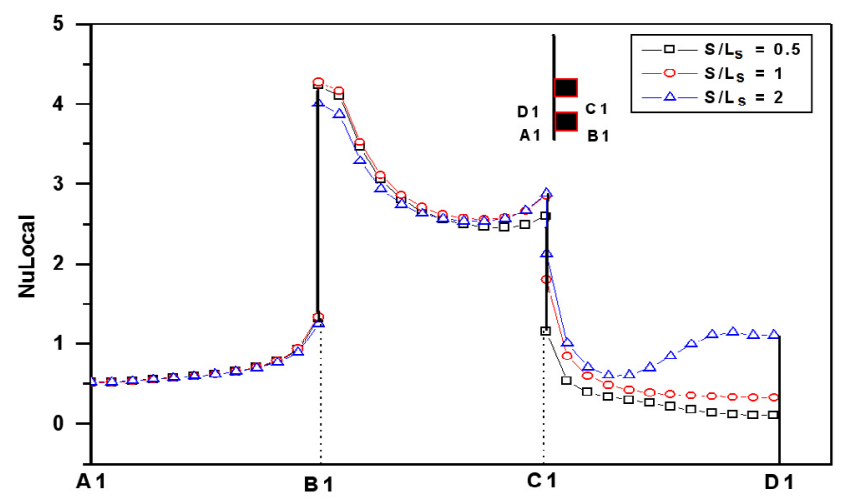

(a) Exposed surfaces of the first heat source $\mathrm{A} 1 \rightarrow \mathrm{B} 1, \mathrm{~B} 1 \rightarrow$ $\mathrm{C} 1, \mathrm{C} 1 \rightarrow \mathrm{D} 1$

Fig. 11. Variation of local Nusselt number $\left(N u_{\text {local }}\right)$ with the distance $(S)$ for the first heat source.

in the tip, where the curves are characterized by an increase in heat transfer in this region when $S=L_{S}$, and $S=2 \times L_{S}$. Finally, on the face C1-D1, the curves of $N u_{\text {local }}$ are completely separate and are characterized by the increase in heat dissipation when $S=2 \times L_{S}$ compared to two other cases that regress. In other words, the total evolution of the local Nusselt number between the two extreme values of $S$ is nearly $67 \%$; however, in terms of intensity of heat dissipation, we note that it will be at its maximum at B1-C1surface. On the three other faces of the second component (Figs. 12b-12d), we find that the same curve profile is visible on each side, however, the intensity of heat transfer is more important and varies when $S=2 \times L_{S}$, mainly, on the face A2-B2 which means that the total Nusselt number $\mathrm{Nu}$ increases significantly (about $7 \%$ ) if we double the space between the heat sources and about $17.8 \%$ when $S \times 4$ (Fig. 13 ). In short, increasing the distance between the components promotes the transfer 


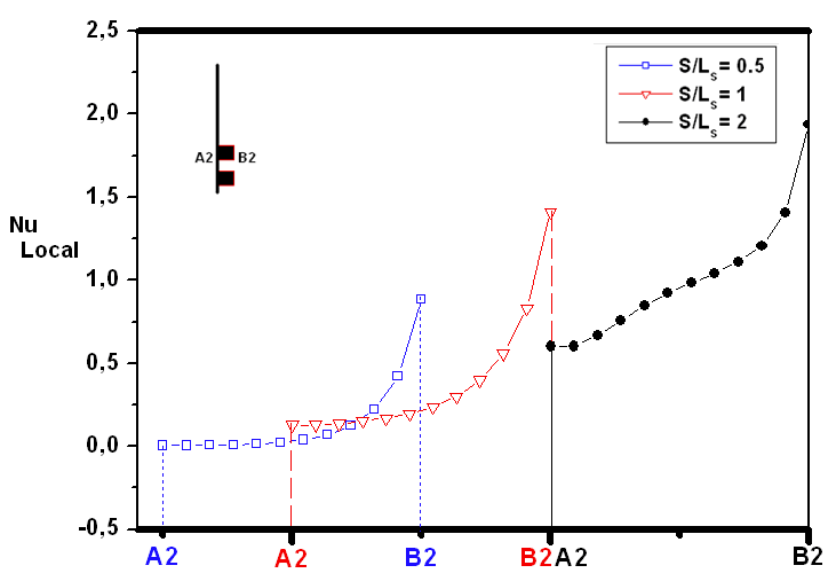

(b) Exposed surface of the $2^{\text {nd }}$ heat source A2 $\rightarrow$ B2

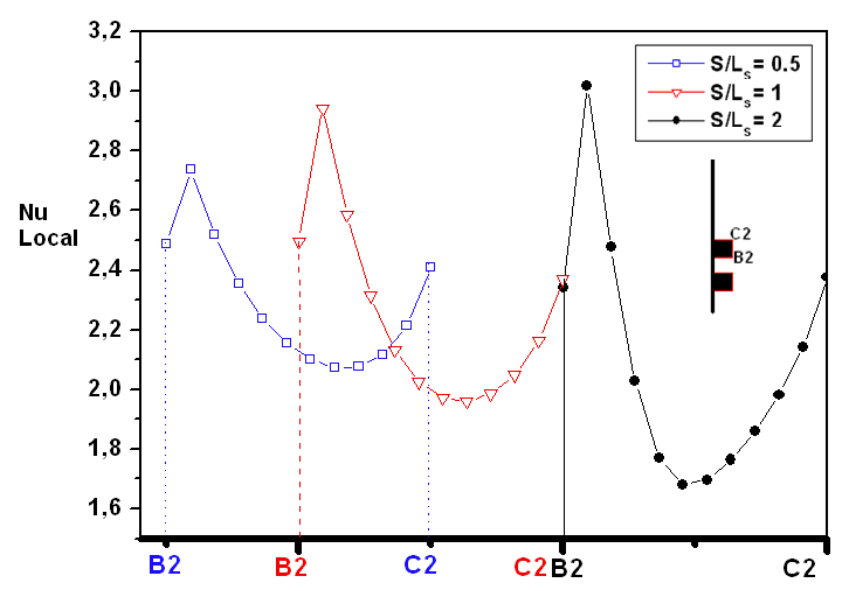

(c) Exposed surface of the $2^{\text {nd }}$ heat source $\mathrm{B} 2 \rightarrow \mathrm{C} 2$

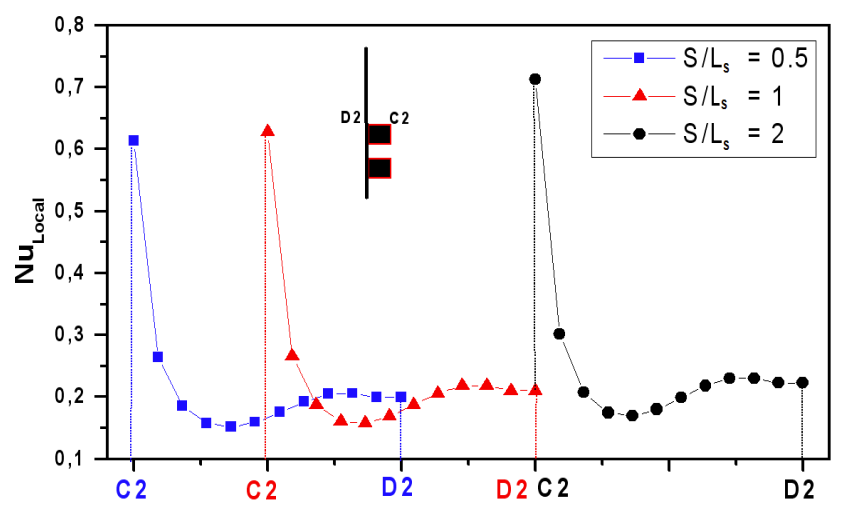

(d) Exposed surface of the of the $2^{\text {nd }}$ heat source $\mathrm{C} 2 \rightarrow \mathrm{D} 2$

Fig. 12. Variation of local Nusselt number $\left(N u_{\text {local }}\right)$ with the distance $(S)$ for the second heat source.

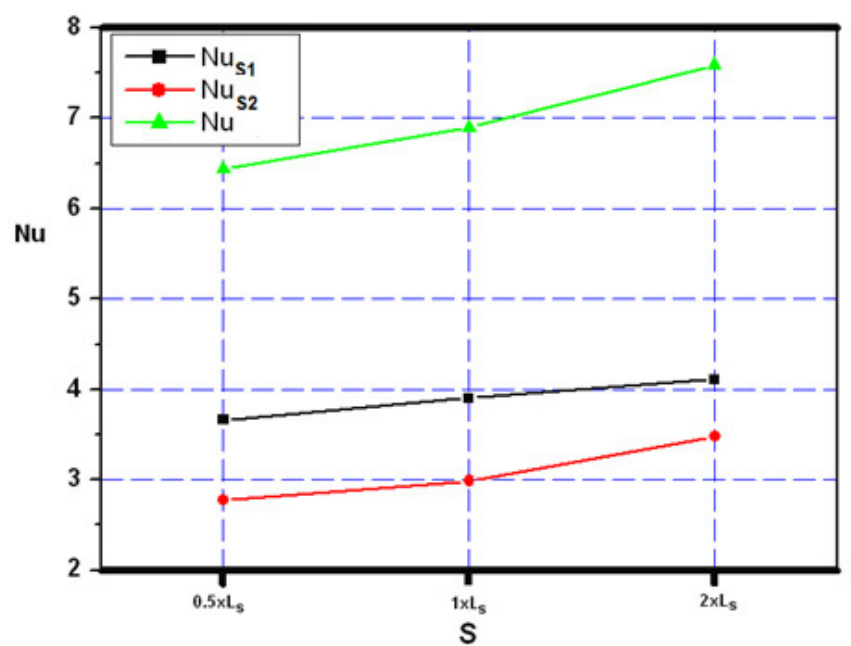

Fig. 13. Variation of total Nusselt number $(N u)$ depending distance between the components $(S)$ for $G r=10^{4}$.

of heat in each component; but, the heat transfer is better at the first component compared to that recorded at the second one.

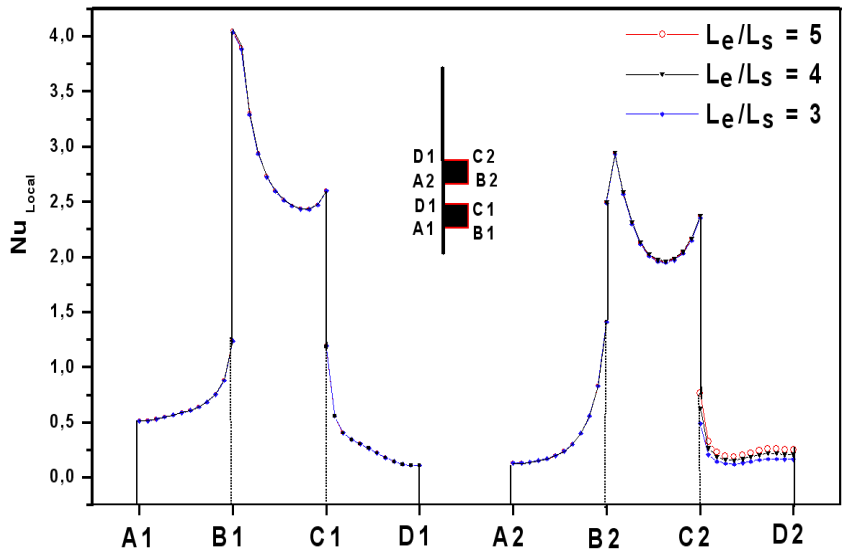

Fig. 14. Effect of the local Nusselt number $\left(N u_{\text {local }}\right)$ with exit distance $\left(L_{\mathrm{e}}\right)$ on each exposed surface of components.

\subsection{Effect of the length of the exit $L_{e}$}

By varying the exit length on Nusselt number, we took the following values: $L_{\mathrm{e}}=3 \times L_{S}, L_{\mathrm{e}}=4 \times L_{S}$, $L_{\mathrm{e}}=5 \times L_{S}$. The data used in this context are: $G r=10^{4}$ and $S=L_{S}$. Figure 14 shows the evolution of the local Nusselt number depending on the length of the output on the six faces of electronic components. On the surfaces 


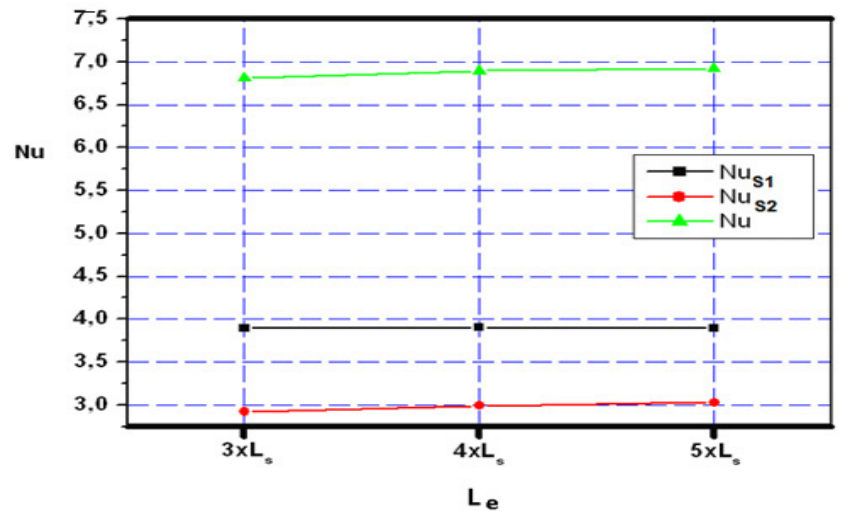

Fig. 15. Variation of total Nusselt number $(N u)$ with the exit length $\left(L_{e}\right)$ for $G r=10^{4}$.

B1-C1 and B2-C2 of the two components, maximum values of $N u_{\text {local }}$ are reached. On these surfaces there is a significant heat transfer, unlike on the other surfaces where the curves are almost identical and $N u_{\text {local }}$ variation is negligible except on the surface C2-D2, where heat transfer is closely proportional to the increase in the distance of $\left(L_{\mathrm{e}}\right)$. The total Nusselt number of the two sources (Fig. 15) increases by $1.6 \%$ when $L_{\mathrm{e}}$ increases from $3 \times L_{S}$ to $5 \times L_{S}$. Therefore, increasing the exit distance increases the heat transfers, primarily for the second component. This conclusion is in agreement with that obtained by Icoz and Jaluria [9].

\section{Conclusion}

The cooling by natural convection of electronic components mounted on vertical and adiabatic wall has been studied numerically by using the finite volume method to solve the equations of the mathematical model.

The results show that a regular and uniform heat sources distribution in the inlet is very important in order to take advantage from the leading boundary layer formed before the first heat source in order to obtain the necessary heat dissipation for an optimal cooling.

The parameters impact on the heat dissipation, characterized by the Nusselt number, has different importance. In fact, the local Nusselt number of the first component is always higher than that of the second because the boundary layer is directly in contact with it. For a Prandtl number $\operatorname{Pr}=0.71$, the $(G r)$ increases the heat exchange that is reflected by an increase of Nusselt number, and also participates in the formation of recirculation zones. The total Nusselt number $\mathrm{Nu}$ is increased when $(G r)$ is multiplied by $10^{2}$. Concerning the distance $(S)$ between the heat sources, the results show that $\mathrm{Nu}$ increases about $7 \%$ if the distance $(S)$ doubles, and becomes approximately $17.8 \%$ when $(S)$ quadruples. At the end, the heat transfer increases when increasing the distance (Le) of the upper exit length, especially on the second component. The total Nusselt number increases by $1.6 \%$ when $(L e)$ increases by about $67 \%$.

The best configuration for which we obtain optimal heat dissipation $\left(N u_{\max }\right)$ is when the Grashof number
$G r$ is important and in which a regular and uniform heat source at the entrance of the flow is provided. Thus, the parameters that have considerable influence on heat transfer are:

1- The intensity of the buoyancy forces characterized by the Grashof number.

2- The length space $S$ between heat sources.

3- The exit length $L_{\mathrm{e}}$.

Acknowledgements. A special thanks to Professor M. El Ganaoui (IUT Longwy) and MrsV. Dawson (F.P Company) for their precious help and patience.

\section{References}

[1] L.T. Yeh, Review of Heat Transfer Technologies in Electronic Equipment, ASME Journal of Electronics Packaging 117 (1995) 333-339

[2] M. Behnia, A.A. Dehghan, H. Mishima, W. Nakayama, Convection Cooling of a Heated Obstacle in a Channel, Int. J. Heat Mass Transfer 41 (1998) 3131-3148

[3] H. Wang, F. Penot, J. Saulnier, Numerical Study of a Buoyancy-induced Flow Along a Vertical Plate with Discretely Heated Integrated Circuit Packages, Int. J. Heat Mass Transfer 40 (1997) 509-1520

[4] L.F. Ramón, O. Berbakow, Natural Convection in Cubical Enclosures with Thermal Sources on Adjacent Vertical Walls, Numerical Heat Transfer, Part A 4 (2002) $331-340$

[5] T.J.R. Dias, L.F. Milanez, Natural Convection Due to a Heat Source on a Vertical Plate, Int. J. Heat Mass Transfer 47 (2004) 1227-1232

[6] A.K. da Silva, S. Lorenzini, A. Bejan, Distribution of Heat Sources in Vertical Open Channels with Natural Convection, Int. J. Heat Mass Transfer 48 (2005) $1462-1469$

[7] R. Comunelo, S. Güths, Natural Convection at Isothermal Vertical Plate, neighborhood influence, 18th International Congress of Mechanical Engineering, November 6-11, Ouro Preto, MG. 2005

[8] H. Bhowmic, K. Tou, Experimental Study of Transient Natural Convection Heat Transfer from Simulated Electronic Chips, Exp. Thermal Fluid Sci. 29 (2005) 485-492

[9] T. Icoz, Y. Jaluria, Numerical Simulation of Boundary Conditions and the Onset of Instability in Natural Convection Due to Protruding Thermal Sources in an Open Rectangular Channel, Numerical Heat Transfer, Part A 48 (2005) 831-347

[10] S. Nadar, Natural Convection Heat Transfer in Horizontal and Vertical Closed Narrow Enclosures with Heated Rectangular Finned Base Plate, Int. J. Heat Mass Transfer 50 (2006) 667-679

[11] J. Harvest, A. Fleischer, R. Weinstein, Modeling of the Thermal Effects of Heat Generating Devices in Close Proximity on Vertically Oriented Printed Circuit Boards for Thermal Management Applications, Int. J. Thermal Sci. 46 ( 2007) 253-261

[12] A. Bejan, Convection heat transfer, 3rd edition, John Wiley and Sons, New York, 2004

[13] S.V. Patankar, Numerical heat transfer and fluid flow, Hemisphere, Washington. DC, 1980 